\title{
Manifestação da Doença de Kawasaki sob espectro clínico da Covid-19: uma revisão sistemática de literatura
}

\author{
Manifestation of Kawasaki Disease under the Covid-19 Clinical Spectrum: a systematic literature \\ review
}

Manifestación de la enfermedad de Kawasaki bajo el espectro clínico de Covid-19: una revisión sistemática de la literatura

\author{
Bárbara Queiroz de Figueiredo \\ ORCID: https://orcid.org/0000-0003-1630-4597 \\ Centro Universitário de Patos de Minas, Brasil \\ E-mail: barbarafigueiredo@unipam.edu.br \\ Ana Luísa Mota \\ ORCID: https://orcid.org/0000-0001-7991-9455 \\ Centro Universitário de Patos de Minas, Brasil \\ E-mail: anamota@unipam.edu.br \\ Francyele dos Reis Amaral \\ ORCID: https://orcid.org/0000-0003-3063-6838 \\ Centro Universitário de Patos de Minas, Brasil \\ E-mail: francyeleamaral@unipam.edu.br \\ Júlia Fernandes Nogueira \\ ORCID: https://orcid.org/0000-0002-5096-1796 \\ Centro Universitário de Patos de Minas, Brasil \\ E-mail: juliafernandes@unipam.edu.br \\ Thainá Gabrielle Miquelanti \\ ORCID: https://orcid.org/0000-0001-7457-2050 \\ Centro Universitário de Patos de Minas, Brasil \\ E-mail: thainagm@unipam.edu.br \\ Francis Jardim Pfeilsticker \\ ORCID: https://orcid.org/0000-0001-6740-194X \\ Centro Universitário de Patos de Minas, Brasil \\ E-mail: francis@unipam.edu.br
}

\begin{abstract}
Resumo
Introdução: Em crianças acometidas pela Covid-19, o quadro de hiperinflamação apresentado demonstrou alterações nas artérias coronárias, característica da doença de Kawasaki (DK). As crianças apresentam febre alta e persistente, erupções cutâneas, edema nas extremidades, conjuntivite não purulenta, dor abdominal, vômitos e diarreia. Objetivo: apresentar as evidências científicas disponíveis até o momento, que trazem uma associação existente entre a Doença de Kawasaki Covid-19 em crianças. Metodologia: Trata-se de uma revisão de literatura realizada entre os meses de junho e julho de 2021, nas seguintes bases de dados: Biblioteca Virtual em Saúde (BVS), Google Scholar, Scientific Electronic Library Online (SciELO) e National Library of Medicine (PubMed MEDLINE). Resultados e discussão: Sugere-se que o mecanismo da DK seja baseado em uma reação pós-inflamatória mediada por anticorpos, assim, a identificação da replicação viral não se mostrou verdadeiramente relevante nesses casos. Além da ligação da pandemia de Covid-19 e aumento dos casos de DK, outro período em que houve pico de incidência da DK foi durante o contexto da pandemia de H1N1, sugerindo que o SARS-CoV-2 não é o único vírus capaz de desencadear tal surgimento da doença de Kawasaki. Conclusão: Apesar das manifestações mais graves nos pacientes acometidos pelo Covid-19, a ocorrência da DK associada à Covid-19 não é muito frequente, no entanto, é importante estar atento aos sinais e sintomas da doença para se obter o diagnóstico mais precoce possível e proceder com o tratamento adequado, de forma a se evitar a ocorrência de quadros clínicos complicados ou agravados.
\end{abstract}

Palavras-chave: Covid-19; Doença de Kawasaki; Doença multissistêmica; Crianças.

\begin{abstract}
Introduction: Children affected by Covid-19, the picture of hyperinflammation presented showed changes in the coronary arteries, characteristic of Kawasaki disease (KD). Children present with persistent high fever, rash, swelling of the extremities, non-purulent conjunctivitis, abdominal pain, vomiting, and diarrhea. Objective: to present the scientific evidence available so far, which brings an existing association between Kawasaki Covid-19
\end{abstract}


Disease in children. Methodology: This is a literature review carried out between June and July 2021, in the following databases: Virtual Health Library (VHL), Google Scholar, Scientific Electronic Library Online (SciELO) and National Library of Medicine (PubMed MEDLINE). Results and discussion: It is suggested that the mechanism of $\mathrm{KD}$ is based on an antibody-mediated post-inflammatory reaction, thus, the identification of viral replication was not truly relevant in these cases. In addition to the link between the Covid-19 pandemic and the increase in DK cases, another period where there was a peak in DK incidence was during the context of the H1N1 pandemic, suggesting that SARS-CoV-2 is not the only virus capable of trigger such emergence of Kawasaki disease. Conclusion: Despite the more severe manifestations in patients affected by Covid-19, the occurrence of KD associated with Covid-19 is not very frequent, however, it is important to be aware of the signs and symptoms of the disease in order to obtain the earliest possible diagnosis. and proceed with the appropriate treatment, in order to avoid the occurrence of complicated or aggravated clinical conditions.

Keywords: Covid-19; Kawasaki Disease; Multisystemic disease; Kids.

\begin{abstract}
Resumen
Introducción: Niños afectados por Covid-19, el cuadro de hiperinflamación presentado mostró cambios en las arterias coronarias, característicos de la enfermedad de Kawasaki (KD). Los niños presentan fiebre alta persistente, erupción cutánea, hinchazón de las extremidades, conjuntivitis no purulenta, dolor abdominal, vómitos y diarrea. Objetivo: presentar la evidencia científica disponible hasta el momento, que trae una asociación existente entre la Enfermedad de Kawasaki Covid-19 en niños. Metodología: Se trata de una revisión de la literatura realizada entre junio y julio de 2021, en las siguientes bases de datos: Virtual Health Library (BVS), Google Scholar, Scientific Electronic Library Online (SciELO) y National Library of Medicine (PubMed MEDLINE). Resultados y discusión: Se sugiere que el mecanismo de KD se basa en una reacción posinflamatoria mediada por anticuerpos, por lo que la identificación de la replicación viral no fue realmente relevante en estos casos. Además del vínculo entre la pandemia de Covid-19 y el aumento de casos de DK, otro período en el que hubo un pico en la incidencia de DK fue durante el contexto de la pandemia $\mathrm{H} 1 \mathrm{~N} 1$, lo que sugiere que el SARS-CoV-2 no es el único virus capaz de desencadenar tal aparición de la enfermedad de Kawasaki. Conclusión: A pesar de las manifestaciones más graves en pacientes afectados por Covid-19, la ocurrencia de EK asociada a Covid-19 no es muy frecuente, sin embargo, es importante estar atento a los signos y síntomas de la enfermedad para poder obtener la diagnóstico lo más temprano posible y proceder con el tratamiento adecuado, con el fin de evitar la aparición de condiciones clínicas complicadas o agravadas.
\end{abstract}

Palabras clave: Covid-19; La enfermedad de Kawasaki; Enfermedad multisistémica; Niños.

\title{
1. Introdução
}

A Doença de Kawasaki (DK) pode ser compreendida como uma vasculite sistêmica primária que acomete pequenos e médios vasos, principalmente as artérias coronárias (Campos et al., 2020). A doença afeta de forma mais frequente crianças, menores que 5 anos e o seu tratamento é indispensável, visto que pode acarretar diversas consequências e até a morte (Gonçalves et al., 2020). Outrossim, o quadro clínico da DK envolve, obrigatoriamente, a presença de febre por pelo menos 5 dias associada a outros critérios estabelecidos pela American Heart Association (AHA), que são: alterações da mucosa oral, conjuntivite não exsudativa bilateral, erupção cutânea exantemática, descamação de mãos e pés e linfadenopatia cervical (Chara et al., 2021).

Os primeiros casos da Covid-19, causada pelo SARS-CoV-2, foram registrados na cidade de Wuhan, na China e, desde então, já atingiu mais de 100 milhões de pessoas em todo o mundo (Campos et al., 2021). A doença apresenta uma característica multissistêmica, e que se manifesta em qualquer idade, sendo mais grave em indivíduos idosos e com presença de comorbidades (Volpat et al., 2020). A Sars-CoV-2 nos adultos geralmente afeta o trato respiratório, causando pneumonia e os sintomas mais comumente encontrados são febre baixa, tosse, odinofagia e fadiga, já as crianças apresentam-se como assintomáticas ou uma sintomatologia leve, como febre intermitente, infecção de vias aéreas superiores e pneumonia sem hipoxemia (Santos et al., 2020).

Contudo, após uma análise de crianças acometidas pela Covid-19, o quadro de hiperinflamação apresentado demonstrou alterações nas artérias coronárias, característica da doença de Kawasaki (Volpat et al., 2020). As crianças apresentam febre alta e persistente, erupções cutâneas, edema nas extremidades, conjuntivite não purulenta, dor abdominal, vômitos e diarreia (Riphagen et al., 2020). Devido à grande semelhança dos sintomas encontrados, existem algumas dúvidas 
acerca dessa doença, se seria realmente a Doença de Kawasaki ou uma doença semelhante, que recebeu algumas terminologias, como síndrome do tipo Kawasaki (KLS), doença de Kawasaki atípica, doença de Kawasaki incompleta, síndrome hiperinflamatória do tipo Kawasaki induzida por SARS-CoV-2 (síndrome SCiKH) e KawaCOVID-19 (Kabeerdoss et al., 2020).

Ademais, a etiologia da Doença de Kawasaki ainda é desconhecida. Porém, com base nas características, tanto clínicas quanto epidemiológicas, aumentam as suspeitas de que a causa desencadeante seja um agente infeccioso, como os vírus respiratórios (adenovírus, enterovírus, coronavírus) (Alizagar 2020). Além disso, após o surgimento da pandemia do Coronavírus, a incidência da Doença de Kawasaki aumentou consideravelmente, e, por meio dos relatos disponíveis na literatura, é possível assimilar o Sars-CoV-2 a DK (Fang et al., 2021). Vários casos foram divulgados, relatando a probabilidade de haver uma ligação entre Covid-19 e DK, como um relato de 15 pacientes pediátricos no Hospital Mount Sinai, na cidade de Nova York e também relatos de outros estados, como Califórnia e Illinois (Sandhaus et al., 2020).

Assim sendo, é de extrema importância que os aspectos relacionados a essas doenças sejam elucidados, a fim de esclarecer se a Doença de Kawasaki pertence ao mesmo espectro patológico da Covid-19, visto que as características clínicas típicas da DK foram encontradas em pacientes pediátricos diagnosticados com o novo coronavírus (Silveira et al., 2020). Portanto, diante do atual cenário pandêmico da Covid-19 e considerando a relevância do tema, a presente revisão bibliográfica busca apresentar as evidências científicas disponíveis até o momento, que trazem uma associação existente entre a Doença de Kawasaki e o Corona Vírus (Covid-19) em crianças.

\section{Metodologia}

Trata-se de uma revisão integrativa de literatura através de seleção de estudos, que foi feita até o mês de julho do ano de 2021 por meio do levantamento de publicações indexadas nas plataformas Biblioteca Virtual em Saúde (BVS), Google Scholar, Scientific Electronic Library Online (SciELO) e National Library of Medicine (PubMed MEDLINE). Os descritores foram selecionados e organizados com operadores booleanos da seguinte maneira: COVID-19 OR SARS-CoV-2 OR Coronavirus AND Kawasaki Disease OR Pediatric Multisystem Inflammatory Syndrome AND Children OR Adolescent OR Pediatric.

Os critérios de inclusão estabelecidos foram: publicações feitas até julho de 2021, sem restrições de idiomas ou localizações, disponíveis na íntegra de forma online e gratuita e que abordassem o conteúdo integral ou parcialmente. Como critérios de exclusão eliminaram-se artigos não relacionados à temática, Doença de Kawasaki e Covid-19, e que não estivessem disponíveis na íntegra de forma online e gratuita. Dessa forma, totalizaram-se 27 artigos, publicados entre 2020 e 2021.

As principais informações das publicações foram organizadas e sintetizadas em forma de tabela, de acordo com os seguintes critérios: autores e ano, desenho/amostra de estudo e características epidemiológicas. A análise dos dados foi dividida em duas categorias que abordaram a fisiopatologia e o quadro clínico: correlação etiológica e imunológica entre Doença de Kawasaki e Covid-19 e principais achados sintomáticos dos pacientes estudados.

\section{Resultados e Discussão}

Após a seleção dos artigos por meio dos critérios de inclusão e de exclusão, elaborou-se uma tabela (Tabela 1) contendo as principais informações sobre os achados epidemiológicos, que, em seguida, foram analisadas sob fisiopatologia e fatores sintomáticos, baseada nos diversos artigos analisados e no ano de publicação. A tabela foi organizada por ordem crescente, a partir da data de publicação dos artigos. A análise permitiu a percepção de que os estudos sugerem que sintomas semelhantes aos da Doença de Kawasaki (DK) tendem a ocorrer durante ou várias semanas após a infecção por Sars-CoV-2, 
causando doença grave que requer cuidados intensivos e intervenções terapêuticas específicas, devido à inflamação do miocárdio em grande parte dos pacientes estudados.

Tabela 1: Estudos utilizados na Revisão Integrativa e principais características epidemiológicas dos casos apresentados pelas amostras.

\begin{tabular}{|c|c|c|}
\hline AUTORES E ANO & METODOLOGIA & CARACTERÍSTICAS EPIDEMIOLÓGICAS \\
\hline Chara B. S., et al. (2020) & Revisão integrativa de literatura & $\begin{array}{l}\text { Constatou-se, em Bergamo, Itália, entre } 2015 \text { e } 2020 \text {, incidência mensal } \\
30 \text { vezes maior de sintomas de DK durante a pandemia de Covid- } 19 \text {. } \\
\text { Além disso, crianças asiáticas tiveram maior probabilidade de apresentar } \\
\text { a forma típica da doença. }\end{array}$ \\
\hline Santos B. S., et al. (2020) & Revisão integrativa de literatura & $\begin{array}{l}\text { Quanto ao sexo, houve neutralidade entre feminino e masculino, } \\
\text { predomínio no território oriental e em descendentes nipônicos residentes } \\
\text { em outros continentes. A síndrome, quando associada ao SARS-CoV-2, } \\
\text { parece atingir crianças mais velhas. }\end{array}$ \\
\hline $\begin{array}{l}\text { Pacífico D. K. S., et al. } \\
(2020)\end{array}$ & Revisão integrativa de literatura & $\begin{array}{l}\text { Ao contrário da DK clássica, a inflamação sistêmica após a infecção pelo } \\
\text { novo Coronavírus afeta crianças e adolescentes. Outras descobertas } \\
\text { intrigantes são que os pacientes com formas graves são menos } \\
\text { caucasianos do que a frequência esperada em sua população em geral, } \\
\text { sendo muitos da etnia afro-americana ou afro-caribenha. }\end{array}$ \\
\hline Campos L. R., et al. (2020) & Revisão integrativa de literatura & $\begin{array}{l}\text { A síndrome inflamatória multissistêmica pediátrica temporalmente } \\
\text { associada ao SARS-CoV-2 (MIS-C) costuma acometer crianças maiores } \\
\text { de } 5 \text { anos de idade e predominantemente afrodescendentes. }\end{array}$ \\
\hline Fang Y., et al. (2020) & Revisão integrativa de literatura & $\begin{array}{l}\text { Na DK associada à Covid- } 19 \text { houve predominância epidemiológica por } \\
\text { crianças acima de } 5 \text { anos, do sexo masculino e com níveis de ferritina } \\
\text { acima de } 1400 \mathrm{mg} / 1 \text {. }\end{array}$ \\
\hline Xu S., et al. (2020) & Carta ao editor & $\begin{array}{l}\text { É intrigante que nenhum caso de DK relatado ou sintomas semelhantes } \\
\text { foram observados em pacientes pediátricos na China desde o surto de } \\
\text { Covid-19 na China, levantando a possibilidade de retrocesso racial a } \\
\text { suscetibilidade genética e de base pode desempenhar um papel. }\end{array}$ \\
\hline Jones V. G., et al. (2020) & Relato de caso & $\begin{array}{l}\text { A paciente estudada era do sexo feminino, } 6 \text { meses de idade, a termo, } \\
\text { totalmente imunizada, previamente saudável, que apresentou } \\
\text { manifestações da DK e, cinco dias depois, testou positivo para Covid-19. }\end{array}$ \\
\hline Zhang X., et al. (2020) & Revisão integrativa de literatura & $\begin{array}{l}\text { Uma incidência excepcionalmente alta de doença de Kawasaki em } \\
\text { crianças foi relatada em um centro francês para doenças infecciosas } \\
\text { emergentes: } 17 \text { casos em } 11 \text { dias, em contraste com uma média de } 2 \text { casos } \\
\text { por mês em } 2018-2019 \text {. Notavelmente, quase } 60 \% \text { dos pacientes eram } \\
\text { originários da África Subsaariana ou ilhas do Caribe, e } 12 \% \text { da Ásia, } \\
\text { levantando, asso, uma possível predisposição genética. }\end{array}$ \\
\hline $\begin{array}{l}\text { Figueroa E. I. R., et al. } \\
(2020)\end{array}$ & Relato de caso & $\begin{array}{l}\text { O paciente estudado era afro-americano, sexo masculino, de } 5 \text { anos de } \\
\text { idade, anteriormente saudável, infectado por Sars-CoV-2 com } \\
\text { apresentações típicas da DK. }\end{array}$ \\
\hline Akca U. K., et al. (2020) & $\begin{array}{l}\text { Revisão integrativa de casos } \\
\text { clínicos }\end{array}$ & $\begin{array}{l}\text { Na revisão, dentre os pacientes relatados, com manifestações da DK que } \\
\text { estavam infectados por Covid-19, } 80 \text { eram da França, } 17 \text { pacientes da } \\
\text { Itália, } 132 \text { pacientes dos EUA, } 74 \text { pacientes do Reino Unido, } 3 \text { pacientes } \\
\text { de Índia, } 12 \text { pacientes da Espanha, } 1 \text { paciente de Israel e } 1 \text { paciente da } \\
\text { Turquia. }\end{array}$ \\
\hline Toubiana J., et al. (2020) & $\begin{array}{l}\text { Estudo } \\
\text { observacional }\end{array}$ & $\begin{array}{l}\text { No hospital estudado, } 21 \text { crianças e adolescentes (idade média } 7,9 \\
\text { (variação } 3,7-16,6) \text { anos) foram admitidos com características de Doença } \\
\text { de Kawasaki em um período de } 15 \text { dias, com } 12(57 \%) \text { de ascendência } \\
\text { africana. } 19 \text { (90\%) tinham evidências de infecção recente por SARS- } \\
\text { CoV-2 (resultado de RT-PCR positivo em } 8 / 21 \text {, anticorpo IgG positivo } \\
\text { detecção em 19/21). }\end{array}$ \\
\hline
\end{tabular}




\begin{tabular}{|c|c|c|}
\hline $\begin{array}{l}\text { Schroeder A. R., et al. } \\
(2020)\end{array}$ & Carta ao editor & $\begin{array}{l}\text { O surgimento de padrões entre várias cidades aponta para uma possível } \\
\text { associação causal entre o fenômeno DK e Covid-19. Os alertas da Itália e } \\
\text { da França ainda contém poucos dados; a maioria das crianças em Nova } \\
\text { York e Inglaterra não tinham provas de infecção aguda com Covid-19, } \\
\text { embora o teste de sorologia positivo resultou, em alguns pacientes, } \\
\text { sugestão de que a síndrome pode representar uma resposta imune } \\
\text { retardada ao Covid-19. }\end{array}$ \\
\hline Russel M. V., et al. (2020) & Carta ao editor & $\begin{array}{l}\text { Artigos citados descreveram dez casos (sete meninos, três meninas; com } \\
7,5 \text { anos de idade) de um tipo de DK em Bergamo, Itália, no auge da } \\
\text { pandemia no país ( } 18 \text { de fevereiro a } 20 \text { de abril de } 2020) \text {, uma incidência } \\
\text { mensal cerca de } 30 \text { vezes maior do que a observada para a doença de } \\
\text { Kawasaki nos } 5 \text { anos anteriores. Ambas as séries descreveram uma idade } \\
\text { média de apresentação mais velha ( } 11,6 \text { anos na coorte francesa e } 7,5 \\
\text { anos na coorte italiana). }\end{array}$ \\
\hline Kam K. Q., et al. (2020). & Carta ao editor & $\begin{array}{l}\text { Os autores descreveram características de dez crianças com diagnóstico } \\
\text { de doença de Kawasaki ao longo de um período de um mês em abril de } \\
2020 \text {, representando um aumento de } 495 \% \text { em comparação com o } \\
\text { hospital de base taxas de admissão ao longo de um período de } 15 \\
\text { anos. Este aumento acentuado na incidência da doença de Kawasaki } \\
\text { ocorreu } 2 \text { semanas após o pico de admissões por Covid-19 em Paris, } \\
\text { França, ou seja, quando o SARS-CoV-2 era o principal vírus respiratório. }\end{array}$ \\
\hline Ouldali N., et al. (2020) & $\begin{array}{l}\text { Estudo } \\
\text { observacional }\end{array}$ & $\begin{array}{l}\text { Entre } 1 \text { de dezembro de } 2005 \text { e } 20 \text { de maio de } 2020,230 \text { pacientes foram } \\
\text { internados no hospital com incompleta ou completa Doença de Kawasaki } \\
\text { (134 [58\%] meninos, } 96 \text { [42\%] meninas, com idade } 1 \text { mês a } 15,5 \text { anos). O } \\
\text { número médio de hospitalizações por DK estimadas era de } 1,2 \text { por mês. } \\
\text { Em abril de } 2020 \text {, observou-se um aumento significativo no número de } \\
\text { pacientes com DK internados neste centro (seis pacientes por mês; } \\
\text { aumento de } 497 \% \text { ). Resultados semelhantes foram obtidos ao analisar a } \\
\text { taxa de doença de Kawasaki por } 100 \text { hospitalizações ao longo do tempo, } \\
\text { com ocorrência de um pico entre } 15 \text { de abril e } 15 \text { de maio de } 2020 \text {. } \\
\text { Assim, o aumento da doença de Kawasaki hospitalizações começaram } 2 \\
\text { semanas após o primeiro pico do Epidemia de Covid- } 19 \text { na região de } \\
\text { Paris, que ocorreu por volta de } 31 \text { de março a } 1^{\circ} \text { de abril de } 2020 \text {. }\end{array}$ \\
\hline Amirfakhryan, H. (2020) & Carta ao editor & $\begin{array}{l}\text { Crianças com sintomas e sinais de DK diagnosticadas após a pandemia de } \\
\text { Sars-CoV-2 mostram evidências de resposta imunológica ao vírus em } \\
\text { crianças mais velhas, com maior taxa de envolvimento cardíaco e } \\
\text { características de síndrome de ativação macrofágica (MAS). }\end{array}$ \\
\hline Loomba R. S., et al. (2020) & Carta ao editor & $\begin{array}{l}\text { Números do Banco Nacional de Dados de Taiwan demonstraram a } \\
\text { ligação da Covid-19 com a DK em pacientes pediátricos com média de } 5 \\
\text { anos de idade. }\end{array}$ \\
\hline Berhelot J. M., et al. (2020) & Revisão integrativa de literatura & $\begin{array}{l}\text { Sintomas de pacientes com Covid- } 19 \text { como pneumonite intersticial, com } \\
\text { vasculopatia inflamatória e linfopenia acentuada foram relacionados à } \\
\text { incidência instantânea de DK. }\end{array}$ \\
\hline Sandhaus H., et al. (2020) & Carta ao editor & $\begin{array}{l}\text { Um caso publicado em } 7 \text { de abril de } 2020 \text { relatou a coexistência de } \\
\text { positividade para Covid-19 e DK. Em } 28 \text { de abril de } 2020 \text {, surgiram } \\
\text { notícias de novas taxas aumentadas dessa doença grave em pacientes } \\
\text { pediátricos sem condições de saúde prévias. Desde então, várias fontes de } \\
\text { notícias têm relatos dessas duas condições que aparecem } \\
\text { simultaneamente ou em rápida sucessão. Digno de nota é o relato de } 15 \\
\text { possíveis casos de DK em pacientes pediátricos, a maioria com } \\
\text { diagnóstico de Covid-19, no Hospital Mount Sinai, na cidade de Nova } \\
\text { York. Também foram mencionados possíveis casos de crianças com } \\
\text { ambas as condições na Califórnia e em Illinois. }\end{array}$ \\
\hline Alizargar J. (2020) & Carta ao editor & $\begin{array}{l}\text { Estudo italiano encontrou aumento de } 30 \text { vezes na DK após o início da } \\
\text { pandemia de Covid-19. Eles também descobriram que casos mais graves } \\
\text { foram encontrados após esse período e que os pacientes com DK durante } \\
\text { a pandemia eram mais velhos do que antes. }\end{array}$ \\
\hline
\end{tabular}




\begin{tabular}{|c|c|c|}
\hline$(2020)$ & & $\begin{array}{l}\text { apresentaram sorologia positiva, indicando infecção prévia por Sars-CoV- } \\
\text { 2. Além disso, alguns resultados diferiram do grupo comparador de DK } \\
\text { "clássica" por demonstrarem idades mais avançadas de instalação do } \\
\text { quadro, observando-se uma média de } 10 \text { anos. }\end{array}$ \\
\hline Volpat A. T., et al. (2020). & Revisão integrativa de literatura & $\begin{array}{l}\text { Doença de Kawasaki associada ao Sars-CoV-2 foi mais frequente em } \\
\text { crianças asiáticas acima de } 5 \text { anos de idade. }\end{array}$ \\
\hline $\begin{array}{l}\text { Carvalho H. T., et al. } \\
(2020)\end{array}$ & Relato de caso & $\begin{array}{l}\text { Enquanto na DK clássica a maior incidência se dá em menores de cinco } \\
\text { anos, a nova síndrome inflamatória acomete crianças mais velhas ou } \\
\text { adolescentes, com média de idade de } 10 \text { anos }\end{array}$ \\
\hline Lessa A. R., et al. (2021) & Revisão integrativa de literatura & $\begin{array}{l}\text { A DK afeta, na maioria das vezes, crianças menores de cinco anos, porém } \\
\text { evidencia-se uma média de idade superior na associação da doença com } \\
\text { Covid-19. }\end{array}$ \\
\hline $\begin{array}{l}\text { Rodrigues J. F., et al. } \\
(2021)\end{array}$ & Revisão integrativa de literatura & $\begin{array}{l}\text { Observa-se uma epidemiologia fora do padrão já conhecido da doença, } \\
\text { tendo sido observada uma apresentação mais tardia ( } 8-11 \text { anos) e atingir } \\
\text { populações como a afrodescendente e hispânica com mais frequência que } \\
\text { os asiaticos, classicamente a população mais acometida }\end{array}$ \\
\hline Pousa P. A., et al. (2021) & Revisão integrativa de literatura & $\begin{array}{l}\text { Doença de Kawasaki associada ao Sars-CoV-2 foi mais frequente em } \\
\text { crianças asiáticas acima de } 5 \text { anos de idade. }\end{array}$ \\
\hline Bailey L. C., et al. (2021) & Revisão integrativa de literatura & $\begin{array}{l}\text { Na coorte de doenças virais de 2020, } 107 \text { pacientes com DK ( } 41 \% \text { ) foram } \\
\text { submetidos ao teste de SARS-CoV-2 e } 8 \text { desses pacientes ( } 8 \% \text { ) tiveram } \\
\text { resultados positivos no teste. }\end{array}$ \\
\hline
\end{tabular}

Fonte: Autores (2021).

Dados mundiais indicam que Covid-19 tende a ser mais benigno em crianças, o que é evidenciado por uma alta incidência de casos de infecção assintomática ou leve de vias aéreas superiores nessa população. No entanto, estudos recentes têm associado sintomas semelhantes aos de Kawasaki como uma apresentação não clássica da doença por coronavírus em pediatria. Sugere-se que a intensa cascata de citocinas, promovida pela infecção pelo SARS-CoV-2, pode desencadear uma resposta inflamatória multissistêmica como forma de Kawasaki atípica em indivíduos geneticamente predispostos. Sob esse contexto, os pacientes podem desenvolver características clínicas mais graves, com maior predisposição ao envolvimento miocárdico, Síndrome de Ativação Macrófago, e Síndrome de Choque da Doença de Kawasaki (Chara et al., 2020). A manifestação de uma síndrome inflamatória multissistêmica em pacientes pediátricos ainda é pouco frequente, com escassos relatos e estudos no meio científico que esclareçam tal distúrbio. Essa descrição também se torna pouco discutida principalmente ao se considerar sua baixa regularidade de casos em comparação à apresentação patológica da Covid-19 entre adultos e idosos. Além disso, a rara literatura sobre a manifestação, patogênese e virulência da síndrome multissistêmica em crianças e adolescentes contribui para o impasse de se descrever sua ocorrência de forma ampla (Volpat et al., 2020).

\section{- Correlação etiológica e imunológica entre Doença de Kawasaki e Covid-19:}

O primeiro relato de caso de uma criança de 6 meses de idade com doença de Kawasaki e Covid-19 foi publicado nos Estados Unidos em 7 de abril de 2020. Desde esse primeiro relato, os países com surtos de SARS-CoV-2 têm relatado mais casos dessa síndrome, denominada de síndrome inflamatória multissistêmica pediátrica temporalmente associada ao SARSCoV-2 (MIS-C), a qual compartilha características clínicas e laboratoriais da doença de Kawasaki (típica ou incompleta), síndrome de choque tóxico estafilocócico e estreptocócico, sepse bacteriana e síndrome de ativação macrofágica (SMA) (Campos et al., 2020).

Além da ligação da pandemia de Covid-19 e aumento dos casos de DK, outro período em que houve pico de incidência da DK foi durante o contexto da pandemia de H1N1, sugerindo que o SARS-CoV-2 não é o único vírus capaz de 
desencadear tal surgimento da doença de Kawasaki. Estes achados estão em linha com observações anteriores relatando associações entre sazonalidade e surtos de Kawasaki doença, especialmente no Japão, onde epidemias em todo o país foram observados em 1979, 1982 e 1986. Padrões sazonais de doença de Kawasaki também foram observados em muitos outros países, incluindo na Europa e na América do Norte (Oldani et al., 2020).

Sob esse contexto, numerosos vírus foram descritos como possíveis gatilhos de DK, incluindo coronavírus (cepas 229E, HKU1, NL63, OC43), embora raramente identificados. Os vírus do herpes, incluindo HHV-6 e -7, foram relatados com mais frequência como desencadeadores de DK (Berhelot et al., 2020). Desse modo, segundo Loomba et al. (2020), os vírus mais frequentemente associados à DK incluem Influenza, Enterovírus, Adenovírus, Parvovírus, Rhovírus, vírus sincicial respiratório, Varicela, Epstein-Barr, sarampo e dengue. Além disso, dentre os marcadores inflamatórios e reativos elevados, incluem a proteína $\mathrm{C}$ reativa, procalcitonina, ferritina e dímero $\mathrm{D}$.

De acordo com estudo de Zhang, et al. (2020), em 82\% dos casos, anticorpos IgG para SARS-CoV-2 foram detectados, sugerindo uma associação entre o vírus e a DK em crianças. Aliado a isso, segundo Chara et al. (2020), dentre o espaço amostral pediátrico mais prevalente, nota-se que a "tempestade de citocinas", fornecida pela infecção ao Sars-CoV-2, é semelhante à ativação de macrófagos na linfo-histiocitose hemofagocítica induzida por vírus, e se assemelha à SMA, que pode ser um gatilho para uma resposta do tipo Kawasaki, em relação à hipótese de DK derivado de uma resposta imune aberrante a um patógeno em indivíduos geneticamente predispostos.

Portanto, sugere-se que seu mecanismo seja baseado em uma reação pós-inflamatória mediada por anticorpos, assim, a identificação da replicação viral não se mostrou verdadeiramente relevante nesses casos. Outrossim, o aumento de infiltrado plasmático de imunoglobulina A (IgA) e macrófagos no epitélio brônquico, inflamação dos tecidos de forma aguda, como nas artérias coronárias e nos linfonodos, presente na DK, é um padrão semelhante à resposta imune inata e adaptativa contra infecções virais (Pacífico et al., 2020). Em contrapartida, perante os números discrepantes entre os casos asiáticos e ocidentais, embora pouco claras, algumas hipóteses são levantadas, como de que a diversidade genômica do Sars-CoV-2 poderia resultar na apresentação de diferentes antígenos, que poderiam ou não levar à ativação do sistema imunológico, resultando em uma tempestade de citocinas. Além disso, diante da variação genética individual nos três principais complexos de histocompatibilidade (MHC) genes de classe 1 que foram relatados nos estudos, pode afetar a gravidade da infecção por SARS-CoV-2, o que também pode explicar as manifestações da DK observadas em algumas crianças da Europa e nos EUA, que poderiam compartilhar um gene MHC de classe 1 diferente, por exemplo, do MHC das crianças asiáticas (Kam et al., 2020).

Assim, crianças afetadas por Covid-19, com suscetibilidade genética a DK, poderiam ter subexpressão genética do receptor ACE2, que pode diminuir ainda mais a expressão de ACE2 devido ao feedback negativo do receptor pelo vírus nesses pacientes, bem como o TNF- $\alpha$ pode ser a causa e a consequência desse feedback ao receptor ACE2. Ou seja, geneticamente, a subexpressão do receptor ACE2 em crianças com suscetibilidade genética a DK que estão infectados com SARS-CoV-2, possivelmente, desregula ainda mais a expressão de ACE2 pelo TNF- $\alpha$, e leva a um aumento repentino de inflamação, incluindo TNF- $\alpha$ e progressão para a DK (Amirfakhryan, 2020).

\section{- Principais achados sintomáticos dos pacientes estudados:}

De acordo com estudo de Santos et al. (2020), dentre as manifestações clínicas relatadas pela amostra, as mais características foram eritema, fissura ou descamação, presentes em 100\% daqueles com alterações dos lábios ou da cavidade oral, enquanto "língua de morango" representou 16,7\%. Em relação às alterações de extremidades, 55,6\% exibiram eritema ou endurecimento firme e 60,7\% edema de mãos e pés. Além disso, febre por cinco dias ou mais foi encontrada em todos os pacientes da revisão. O segundo sintoma mais prevalente foi conjuntivite não exsudativa (83,3\%), seguida por erupção cutânea 
polimórfica (75\%), alterações dos lábios ou da cavidade oral (58,3\%), alterações de extremidades (56,3\%) e linfadenopatia cervical (29,2\%). Apresentações dermatológicas foram definidas como erupções cutâneas, descamação perineal ou facial, eritemas e exantemas, bem como manifestações oculares, como secreção ocular lacrimejante, conjuntivite e eritema periorbital (Pousa et al., 2021). Além disso, segundo Silveira, et al. (2020), esses pacientes tendem a evoluir com envolvimento respiratório e gastrointestinal, sinais meníngeos e de envolvimento cardiovascular, manifestações essas compartilhadas por portadores de Covid-19.

Segundo relato de caso exposto por Jones et al. (2020), a paciente foi atendida na emergência pediátrica com febre, agitação e recusa em aceitar alimentação. No segundo dia de febre, ela desenvolveu uma erupção na pele eritematosa e, no quarto dia, erupção cutânea persistente e possível congestão leve, mas sem tosse. Além desses sintomas, a criança apresentava irritabilidade, conjuntivite poupadora de limbo, e lábios secos. No quinto dia de febre, a criança permaneceu com conjuntivite poupadora de limbo, além de apresentar papilas proeminentes da língua, maculopapular, polierupção cutânea mórfica, latejante e edema das mãos e extremidades inferiores, atendendo, assim, aos critérios críticos clássicos da DK.

Aliado a isso, relato de caso publicado por Figueroa et al. (2020), expôs o caso de uma criança, infectada por Covid19, de 5 anos de idade, que foi admitida no andar de internação pediátrica com febre diária de $39,4^{\circ} \mathrm{C}$ por 8 dias, com história de erupção cutânea, inchaço (palmas das mãos e plantas dos pés), conjuntivite, diminuição do apetite, diarreia, disúria e abdominal dor. O exame físico mostrou seco, lábios rachados e eritematosos, conjuntivite não exsudativa, e linfadenopatia cervical aguda bilateral, mas não irritação na pele. Ele tinha edema escrotal à direita e hidrocele sugestivo de epididimo-orquite à ultrassonografia. Clinicamente, ele atendeu aos critérios para Kawasaki incompleto doença.

Uma série de relatos de caso, dentre crianças de 2 a 10 anos, presente em estudo de Akca et al. (2020), apresentaramse, na maioria dos pacientes, com manifestações sintomáticas como febre, tosse, erupção eritematosa, hiperemia da mucosa oral, vômitos, linfadenopatia. A maioria dos sintomas relatados pelos pacientes da amostra foram dor de cabeça, tosse, coriza, febre por menos de 48 horas e, para um paciente, anosmia. A mediana duração entre esses sintomas e o início de sinais e sintomas da doença de Kawasaki foi de 45 (intervalo de 18 a 79) dias (Toubiana et al., 2020).

Foi observado que Covid-19 danifica o miocárdio, e que maior proteção cardíaca pode ser importante para aqueles com doenças cardíacas preexistentes. Sendo a DK uma uma vasculite que afeta o coração, ela pode ter maior implicação cardíaca na presença de Covid-19. Dessa forma, atrasos no diagnóstico de DK podem multiplicar esse fenômeno em maior capacidade do que sem os efeitos cardíacos da Covid-19 (Sandhaus et al., 2020). A maioria das crianças se recupera completamente de DK após algumas semanas, mas o tratamento precoce é necessário para prevenir possíveis complicações, haja vista que uma forma grave de complicações é coronária aneurismas de artéria, cuja ruptura leva à formação de trombose e infarto do miocárdio (Xu, et al., 2020). A gravidade da doença relatada em alguns desses pacientes aumenta a necessidade de uma definição de caso concreto, que pode ajudar, com o reconhecimento precoce, o tratamento adequado (Schroeder et al., 2020).

Curiosamente, um estudo de coorte de mais de 135 mil pacientes pediátricos com Covid-19, publicado por Bailey et al., (2021), indicou um menor número de diagnósticos de DK feitos durante a pandemia Covid-19 começando no início de 2020 como em comparação com 2018-2019. Isso pode ser um reflexo de uma redução real no número de casos de DK devido às medidas de distanciamento social e hábitos de higiene pessoal promovidos durante a pandemia, o que provavelmente reduziu a transmissão de agentes infecciosos associados a DK. No entanto, pode haver uma subnotificação de síndrome semelhante a Kawasaki em Covid-19 pediátrico devido à falta de consciência e reconhecimento, como bem como acesso reduzido aos cuidados de saúde durante a pandemia, levando a diagnósticos perdidos e, portanto, uma subestimação da verdadeira prevalência da prevalência de DK e Covid-19, instantaneamente. 
$\mathrm{O}$ reconhecimento desta doença no final da onda pandêmica pode estar relacionado à sua raridade e à dificuldade de reconhecer síndromes incomuns em sistemas de saúde fragmentados se reorganizando rapidamente para lidar com uma pandemia. (Russel et al., 2020). O fato de que como os sintomas da DK se manifestam em associação a uma infecção concomitante de Covid-19 também indicam que é não uma doença por si só, mas sim parte de uma síndrome desencadeada por infecção que pode ser mais grave do que DK tradicional e exige reconhecimento precoce e muito mais tratamento agressivo (Fang et al., 2020). Embora os estudos sobre DK e Covid-19 sejam raros e não possam inferir uma relação de causalidade entre elas, mais estudos podem ser sugeridos neste campo. Porém, devido à importância da apresentação de DK nas crianças e ao fato de que atrasos no diagnóstico e encaminhamento contribuem significativamente para a mortalidade em casos de DK, atenção especial deve ser dada aos casos com sintomas do tipo Kawasaki referentes aos hospitais e clínicas durante epidemias virais como Covid-19 (Alizargar 2020).

A hipótese etiopatogênica mais aceita até o momento é de que a doença de Kawasaki seja desencadeada por agentes infecciosos que determinam manifestações clínicas em indivíduos susceptíveis (Carvalho et al., 2020), sugerindo-se que a família do coronavírus pode representar um dos gatilhos para DK, sendo a. SARS-CoV-2, uma cepa altamente virulenta, causando intensa resposta imune no hospedeiro (Lessa et al., 2020). Desse modo, o reconhecimento da similaridade entre a apresentação da síndrome descrita e a Doença de Kawasaki se torna essencial para o manejo clínico da doença e para o impedimento da progressão grave do quadro, sendo primordial a atuação de uma equipe multidisciplinar pediátrica para o reconhecimento de casos, para a observação de sinais de alerta e para o manuseio correto desses pacientes. Vale ressaltar que, embora as repercussões dessa patologia tragam preocupações em relação a uma manifestação nova, aguda e grave da COVID19 em crianças e adolescentes, felizmente, tais ocorrências são incomuns até o momento (Volpat et al., 2020).

As informações apontam para a ocorrência de uma síndrome pós-viral que pode surgir no período entre duas e quatro semanas após a contaminação pelo vírus, os dados revelam a tendência para sintomas como febre e sintomas gastrointestinais que podem evoluir para quadros mais graves com complicações cardíacas, hipotensão, choque e óbito. Portanto, os impactos causados por essa nova conjuntura ainda são desconhecidos, decisões estratégicas no manejo da pandemia como a volta as aulas devem considerar a possibilidade no aumento do número de casos da DK (Rodrigues et al., 2021). No entanto, embora muitos casos apontem para uma possível associação positiva entre DK e Covid-19, as evidências são insuficientes para provar que as duas condições compartilham uma relação causal neste estágio.

\section{Conclusão}

Os estudos indicam que a SARS-CoV-2 pode ser um gatilho para a Doença de Kawasaki, sendo que a hipótese mais bem aceita dessa associação é de que a alta virulência do vírus desencadeia uma "tempestade de citocinas", semelhante à ativação de macrófagos na linfo-histiocitose hemofagocítica. Em crianças com suscetibilidade genética a DK que estão infectados com SARS-CoV-2, possivelmente, desregula ainda mais a expressão de ACE2 pelo TNF- $\alpha$, e leva a um aumento repentino de inflamação, incluindo TNF- $\alpha$ e progressão para a DK.

As manifestações clínicas da DK associada ao Covid-19 nos pacientes pediátricos envolvem os sistemas respiratório e gastrointestinal, sinais meníngeos e de envolvimento cardiovascular, com apresentação de quadros clínicos mais graves que os relatados na DK não associada ao Covid-19, podendo evoluir para complicações cardíacas, hipotensão, choque e até morte. Além disso, o perfil epidemiológico levantado nos estudos é diferente do perfil tradicional da DK, afetando mais amplamente crianças mais velhas e de outras etnias.

Apesar das manifestações mais grave nos pacientes acometidos pelo Covid-19, a ocorrência da Doença de Kawasaki associada ao novo corona vírus não é muito frequente, no entanto, é importante estar atento aos sinais e sintomas da doença para se obter o diagnóstico mais precoce possível e proceder com o tratamento adequado, de forma a se evitar a ocorrência de 
quadros clínicos complicados ou agravados. Sendo assim, é de suma importância que mais estudos clínicos e empiristas, principalmente, investiguem a relação entre DK e Covid-19, a fim de otimizar o tratamento desses pacientes.

\section{Referências}

Akca, U. K., Kesici, S., Ozsurekci, Y., Aykan, H. H., Batu, E. D., Atalay, E., Demir, S., Sag, E., Vuralli, D., Bayrakci, B., Bilginer, Y. \& Ozen, S. (2020). Kawasaki-like disease in children with COVID-19. Rheumatology, 40 (1), 2105-2115.

Alizargar, J. (2020). The novel coronavirus (COVID-19) and the risk of Kawasaki disease in children. Journal of the Formosan Medical Association, 1-2. https://doi.org/10.1016/j.jfma.2020.05.030

Amirfakhryan, H. (2020). Kawasaki-like disease in children with COVID-19: A hypothesis. Medical Hypotheses, 143 (110117), 1-4. https://doi.org/10.1016/j.mehy.2020.110117

Bailey, L. C., Razzaghi, H., Burrows, E. K., Bruno, C. \& Forrest, C. B. (2020). Assessment of 135794 Pediatric Patients Tested for Severe Acute Respiratory Syndrome Coronavirus 2 Across the United States. JAMA Pediatrics, 175 (2). 176-185.

Campos, L. R., Cardoso, T. M., Martinez, J. C. F. F., Almeida, R. G., Silva, R. M., Fonseca, A. R. \& Sztajnbok, F. R. (2020). Síndrome inflamatória multissistêmica pediátrica (MIS-C) temporalmente associada ao COVID-19. Residência Pediátrica, 10 (2), 1-6.

Carvalho, H. T., Thomazi, L., Fioretto, J. R. \& Carpi, M. F. (2020). Manifestações graves da doença de Kawasaki em tempos de COVID-19: relato de caso. Residência Pediátrica, 10 (3), 1-5.

Chara, B. S., Rickli, J. M., Silva, C. F., Pomaleski, G. \& Gonçalves, M. V. M. (2021). Doença semelhante a Kawasaki, um novo fenótipo em Sars-CoV-2? Int J Cardiovasc Sci, 1-4. https://doi.org/10.36660/ijcs.20200186

Fang, Y., Aravamudan, V. M., Sridharan, G. K. \& Abid, M. B. (2021). Kawasaki like illness due to COVID-19: a review of the literature. J Infect Dev Ctries, 15 (5), 630-638.

Figueroa, E. I. R., Santos, R., Simpson, S. \& Garg, P. (2020). Incomplete Kawasaki Disease in a Child with COVID-19. Indian Pediatrics, 57 (1), 680-681.

Gonçalves, L. F., Gonzales, A. I., Patatt, F. S. A., Paiva, K. M. \& Haas, P. (2020). Kawasaki and COVID-19 disease in children: a systematic review. Rev Assoc Med Bras, 66 (2), 136-142. http://dx.doi.org/10.1590/1806-9282.66.S2.136

Jones, V. G., Milss, M., Suarez, D., Hogan, C. A., Yeh, D., Segal, J. B., Ngyuen, E, L., Barsh, G. R., Maskatia, S. \& Mathew, R. (2020). COVID-19 and Kawasaki Disease: Novel Virus and Novel Case. Hospital Pediatrics, 10 (6), 537-540.

Kaberdoss, J., Pilania, R. K., Karkhele, R., Kumar, T. S. \& Danda, D. (2020). Severe COVID-19, multisystem inflammatory syndrome in children, and Kawasaki disease: immunological mechanisms, clinical manifestations and management. Rheumatology International, 41 (1), $19-32$.

Kam, K. Q., Ong, J. S. M. \& Lee, J. H. (2020). Kawasaki disease in the COVID-19 era: a distinct clinical phenotype? The Lancet, 4 (1), 641-643. https://doi.org/10.1016/ S2352-4642(20)30207-8

Lessa, A. R., Auer, A. C. R., Freitas, E. N., Lessa, G. R. \& Prates, L. S. (2021). Associação de SARS-COV-2 com a doença de Kawasaki. Acervo Saúde, 23 (1), 1-6. https://doi.org/10.25248/REAC.e6462.2021

Loomba, R. S., Villarreal, E. \& Flores, S. (2020). COVID-19 and Kawasaki syndrome: should we really be surprised? Cardiology in the Young, 30 (1), 10591060 .

Ouldali, N., Pouletty, M., Mariani, P., Beyler, C. \& Meinzer, U. (2020). Emergence of Kawasaki disease related to SARS-CoV-2 infection in an epicentre of the French COVID-19 epidemic: a time-series analysis. Lancet Child Adolesc Health, 4642 (20), 30174-30179. https://doi.org/10.1016/S23524642(20)30175-9

Pacífico, D. K. S., Silva, F. D. S., Pinto, A. S. B., Junior, E. F. V. \& Batista, L. O. (2020). Doença de Kawasaki e COVID-19: uma revisão de literatura. Acervo Saúde, 12 (12), 1-8. https://doi.org/10.25248/reas.e5085.2020

Pousa, P. A., Mendonça, T. S. C., Oliveira, E. A. \& Silva, A. C. S. (2021). Extrapulmonary manifestations of COVID-19 in children: a comprehensive review and pathophysiological considerations. Jornal de Pediatria. 97 (2), 116-139.

Riphagen, S., Gomez, X., Martinez, C. G., Wilkinson, N. \& Theocharis, P. (2020). Hyperinflammatory shock in children during COVID-19 pandemic. The Lancet, 395 (10237), 1607-1608. https://doi.org/10.1016/S0140-6736(20)31094-1

Rodrigues, J. F. \& Eulálio, E. J. C. (2021). Ocorrência de condições inflamatórias pediátricas na COVID-19: uma revisão integrativa. Research, Society and Development, 10 (3), 1-15. http://dx.doi.org/10.33448/rsd-v10i3.13242

Russell, M. V. \& Whittaker, E. (2020). Kawasaki-like disease: emerging complication during the COVID-19 pandemic. The Lancet, 395 (1), $1741-1742$.

Sandhaus, H., Crosby, D., Sharma, A. \& Gregory, S. R. (2020). Associação entre COVID-19 e doença de Kawasaki: vigilância exigida de otorrinolaringologistas. Academia Americana de Otorrinolaringologia, 1-2.

Santos, B. S., Santos, F. S. \& Ribeiro, E. R. (2020). Clinical-epidemiological relation between SARS-CoV-2 and Kawasaki disease: an integrative literature. Rev Paul Pediatr, 39 (1), 1-8. http://dx.doi.org/10.1590/1984-0462/2021/39/2020217

Schroeder, A. R., Wilson, K. A. \& Ralston, L. S. (2021). Covid-10 and Kawasaki Disease: Finding the Signal in the Noise. Hospital Pediatrics, 10 (10), 1-5. 
Research, Society and Development, v. 10, n. 11, e468101119852, 2021

(CC BY 4.0) | ISSN 2525-3409 | DOI: http://dx.doi.org/10.33448/rsd-v10i11.19852

Silveira, A. C. B., Maia, M. E. D., Zambeli, L. O., Ruela, V. P., Júnior, L. D. M., Cunha, R. M. C. \& Alvim, V. F. (2020). Síndrome inflamatória multissistêmica pediátrica induzida por SARS-CoV-2 e similaridades com a doença de Kawasaki, HU Revista, 46 (1), 1-9.

Toubiana, J., Poirault, C., Corsia, A., Bajolle, F., Fourgeaud, J., Angoulvant, F., Debray, A., Basmaci, R., Salvador, E., Biscardi, S., Frange, P. \& Chalumeau, M. (2020). Kawasaki-like multisystem inflammatory syndrome in children during the covid-19 pandemic in Paris, France: prospective observational study. BMJ, 369 (2094), 1-7.

Volpat, A. T., Gomes, E. M. S., Cunha, E. T., Vilaça, H. M., Mayrink, L. B., Hilarino, L. V. \& Alvim, A. L. (2020). Síndrome inflamatória multissistêmica em crianças e adolescentes com COVID-19: uma revisão de literatura. Journal of Infection Control, 9 (3), 162-165.

Xu, S., Chen, M. \& Weng, J. (2020). COVID-19 and Kawasaki disease in children. Pharmacological Research, 159 (1), 1-3. https://doi.org/10.1016/j.phrs.2020.104951

Zhang, X., Lei, B, Yuan, Y., Zhang, L., Hu, L., Jin, S., Kang, B., Liao, X., Sun, W., Xu, F., Zhong, Y., Hu, J. \& Qi, H. (2020). Brain control of humoral immune responses amenable to behavioural modulation. Nature, 581 (7807), 204-208. 